\title{
Changes in the mechanical properties and microstructure of anisotropic austenitic stainless sheet steel after uniaxial tensile test
}

\author{
Emil Yankov ${ }^{1, *}$, and Maria Nikolova ${ }^{1}$ \\ ${ }^{1}$ University of Ruse, Department Material Science and Technology, Ruse, 8 Studentska Str., Bulgaria
}

\begin{abstract}
The aim of the investigation is to study the changes in the characteristics of an austenitic sheet material X5CrNi18-10 (1.4301, AISI 304) after a plastic deformation. Samples are cut out from the sheet material at three different directions $-0^{\circ}, 45^{\circ}$ and $90^{\circ}$ angle to the rolling direction. The changes in the mechanical properties and microstructure of the anisotropic austenitic steel are investigated by mechanical tests (uniaxial tension tests and hardness measurements) and structural analyses (optical and scanning electron microscopy, X-ray diffraction). It is established that the strain induced phase transformation of the metastable austenite to martensite during the tension tests changes the magnetic properties of the steel. It is found out that the sheet anisotropy effect on the uniform deformation, the thickness reduction and structure of the austenite sheet material is more essential for the plastic deformation behaviour than the strain-induced $\gamma \rightarrow \alpha^{\prime}$ phase transformation.
\end{abstract}

\section{Introduction}

Many research teams $[1,2]$ are dealing with the problem of the unequal deformation of sheet metal materials during the technological process of manufacturing of different parts. One of the aspects in turns of cold plastic deformation is the unequal thickness reduction during the complex loading schemes that leads to earlier destruction of the material. Another aspect is the instrument friction with the sheet material that impede the equal plastic deformation. Most of the authors are trying to explain this improper depletion in thickness and technological difficulties during plastic deformation with different surveys introduced by one direction cut (usually identical with the rolling direction (RD)) samples [3-6]. This approach does not fully explain the differences in the strain values in the other directions relative to the RD.

The texture of the austenite could not only evolve during the deformation, but also be inherited by the initial rolling. As the microstructure of the stainless steel is composed of metastable austenite $(\gamma)$, the latter during cold plastic deformation transforms from paramagnetic phase into ferromagnetic martensite $\left(\alpha^{\prime}\right)$ that has bodycentered cubic (bcc) lattice and is thought to nucleate at the intersections of the shear bands [7]. The strain induced texture of the austenite and the resultant martensite that is formed during the plastic

*Corresponding author: eyankov@uni-ruse.bg 
deformation is very much influenced by the initial texture (anisotropy) of the material. Then, the enhanced work hardening, that influences the forming process and the final product received, changes the plastic technological behaviour of the sheet material.

In order to determine correctly the mechanical properties and to support the technological processes, a detailed examination of samples cut at three different directions in respect to the $\mathrm{RD}$ is necessary to be performed. In the present study the chosen directions in respect to the $\mathrm{RD}$ are $0^{\circ}, 45^{\circ}$ and $90^{\circ}$. Additionally, for clarification of the differences in the received results, a detailed microstructural analysis that explains the unequal changes during deformation is needed. Therefore, the aim of this study is to define in details the mechanical, structural and phase changes in three different directions with respect to the $\mathrm{RD}$ of the $\mathrm{X} 5 \mathrm{CrNi} 18-10$ austenite sheet material.

\section{Experimental procedures}

The material was received in the form of 0.8 -mm-thick sheets in cold-rolled condition. Sheets with the following dimensions: $1000 \times 2000 \mathrm{~mm}$ and thickness of $\mathrm{t}_{0}=0.8 \mathrm{~mm}$ were cut from metal alloy X5CrNi18-10 (AISI 304, DIN 1.4301, SUS 304, GOST 07Ch18N10) with chemical composition shown in Table 1, measured by quantometer SpectroMaxx.

Table 1.Chemical composition of X5CrNi18-10 steel.

\begin{tabular}{|c|c|c|c|c|c|c|c|c|c|c|}
\hline Element & $\mathbf{C}$ & $\mathbf{S i}$ & $\mathbf{M n}$ & $\mathbf{N i}$ & $\mathbf{C r}$ & $\mathbf{M o}$ & $\mathbf{T i}$ & $\mathbf{S}$ & $\mathbf{P}$ & $\mathbf{F e}$ \\
\hline Wt.\% & 0.055 & 0.54 & 1.13 & 7.77 & 18.46 & 0.282 & 0.0042 & 0.0078 & 0.037 & Rest \\
\hline
\end{tabular}

Samples are cut out from the sheet material at three different directions $-0^{\circ}, 45^{\circ}$ and $90^{\circ}$ to the $\mathrm{RD}$ as shown in Fig. 1a. Specimens used for the mechanical properties measurements were determined on the base of standard ISO 6892-1:2009 (Fig. 1 a and b) and cut from the sheet with digital laser guillotine. The material was tested in as-received (called undeformed) conditions.
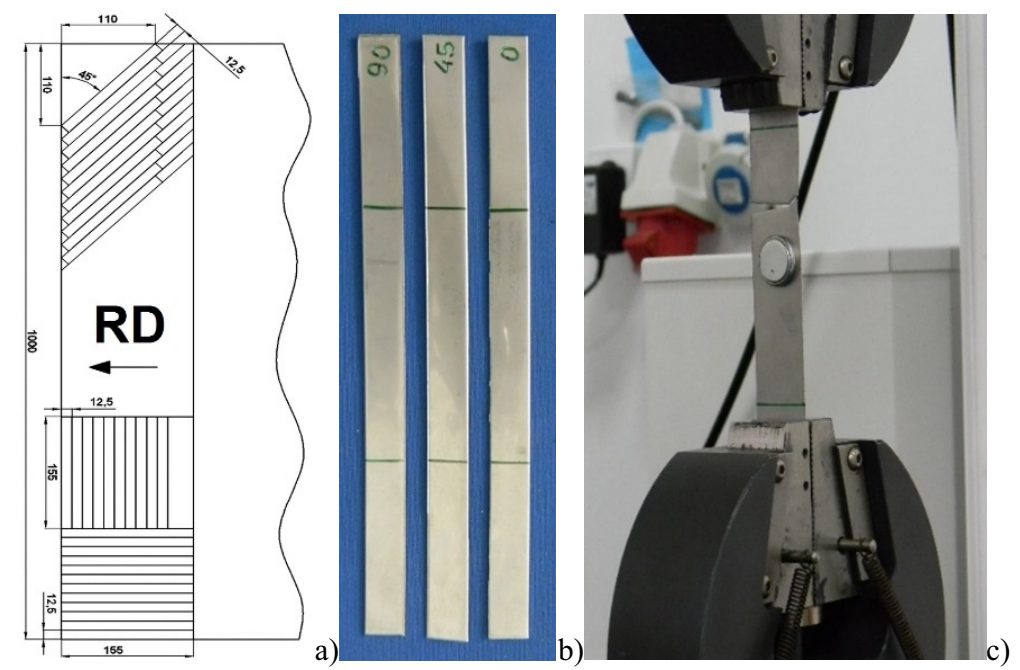

Fig. 1. Sample preparation and testing: a) a scheme of cutting of the samples; b) overall appearance of the samples; c) a fixed and tensile tested sample with the machine. 
The static uniaxial tensile test was performed on a universal testing machine Instron3384, supplied with a software Bluehill-3 at ambient temperature. For the purpose of the testing method a constant strain rate of $5 \mathrm{~mm} / \mathrm{min}$ until the load fell down to $100 \mathrm{~N}$ (or the maximum load fell below 90\%) was used. For each test a control measurement of the working length of testing, height and width was held and the values were set in the used test method via the software program. The data were saved in an appropriate digital form for subsequent analysis.

For the microstructure analysis and hardness measurements three mutually perpendicular planes from each specimens cut near the break down zone were prepared. This zone was chosen in order to monitor and analyse the maximal change in the structure and properties after the deformation. The microstructure and hardness of tensile tested samples were compared to the undeformed ones. Each sample (undeformed and tensile tested (TT)) was evaluated in this three different directions: 1) alongside the long side of the sample (plane I); 2) cross-section (plane II) and 3) top view (plane III). The surface preparation for microstructure analysis and hardness measurements of all sections of the samples before and after the tensile test involved grinding with paper of grit P400, 500, $800,1000,1500,2500$ and mechanical polishing with non-abrasive paste.

A Vickers Hardness tester 432 SVD by Wilson-Wilpert was used for the hardness measurements of the steel according to the standard ISO 6507-1. In order to compare the hardness changes the researches were made on the plane I, II and III of each sample (undeformed and TT) with a load of $5 \mathrm{~kg}$ and dwell time of $10 \mathrm{~s}$. Ten sequential measurements by increments of $50 \mu \mathrm{m}$ between the imprints were made for each plane. Consequently, the data received were gathered in tables for comparative analysis. The summary results of the hardness measurements were presented in graphical form.

Optical microscopy (Epytip, Zeiss, Jena) was performed on the cross sections and top views after etching the samples with aqua regia. To ensure the comparability of the survey results for each plane of all samples one and the same magnification was utilized. 8megapixel digital camera was adapted to the microscope and used for the image acquisition. The micrographs were process by Mountains Map premium v.7 software.

A JEOL JXCA-733 Microprobe Scanning Electron microscope (SEM) was used to study the austenite deformed grains and martensite morphology. The focused electron beam for image acquisition was operated at $19.9 \mathrm{kV}$ at magnification between $600 \times$ and $1000 \times$.

The X-ray phase identification was performed with URD-6 diffractometer, applying BraggBrentano geometry and $\mathrm{Fe}-\mathrm{K} \alpha$ radiation and working at $30 \mathrm{kV}$ and $20 \mathrm{~mA}$. The diffraction angle range scanned was $40-129^{\circ} 2 \theta$ with a step size of $0.1^{\circ} 2 \theta$ and counting time of 5 $\mathrm{s} / \mathrm{step}$. The lattice strain response was measured in the longitudinal and transverse directions of plane III of all samples. The qualitative phase analysis was held using Match!3 software.

The texture analysis was performed by calculating the texture coefficient [8] measuring the relative degree, of preferential orientation among the crystal planes:

$$
T C_{(h k l)}=\frac{\frac{I_{(h k l)}}{I_{0}(h k l)}}{\frac{1}{N} \sum_{(h k l)}^{I_{(h k l)}}}
$$

where I (hkl) and Io (hkl) are the measured intensity and standard integrated intensity (from $\mathrm{PDF}$ ) for (hkl) reflection, respectively, and $\mathrm{N}$ is the number of reflections observed. The data are taken from the results process by the Match software. A TC $>1$ indicates preferential orientation/texture. 


\section{Experimental results}

The representative indicator diagrams (Fig. 2a) are result of five samples testing from one the same angle of cutting sample $\left(0^{\circ}, 45^{\circ}\right.$ and $90^{\circ}$, respectively). The experimentally determined data indicate the load at maximal charge $\mathrm{Fm}$ for tensile extension at maximum charge $\Delta \mathrm{Lm}$, elongation at break $\Delta \mathrm{Lbr}$, elongation at tensile strength at allongement $\mathrm{A}$ and tensile strength $\mathrm{Rm}$. The diagram considered as representative is the one with the closest mechanical properties to the average results from each indicator diagram for a particular angle of cutting [9]. The highest Fm values occurs within the $45^{\circ}$ angle of cutting samples - Fm $=6.153 \mathrm{kN}$ that is $15 \%$ higher than the $90^{\circ}$ and $18 \%$ higher than the $0^{\circ}$ tested samples. The same trend is observed with elongation at break $\Delta \mathrm{Lbr}$ of the $45^{\circ}$ cut sample $-\Delta \mathrm{Lm}=24.934 \mathrm{~mm}$ that is $51 \%$ and $73 \%$ higher than the $90^{\circ}$ and $0^{\circ}$ tested samples, respectively.
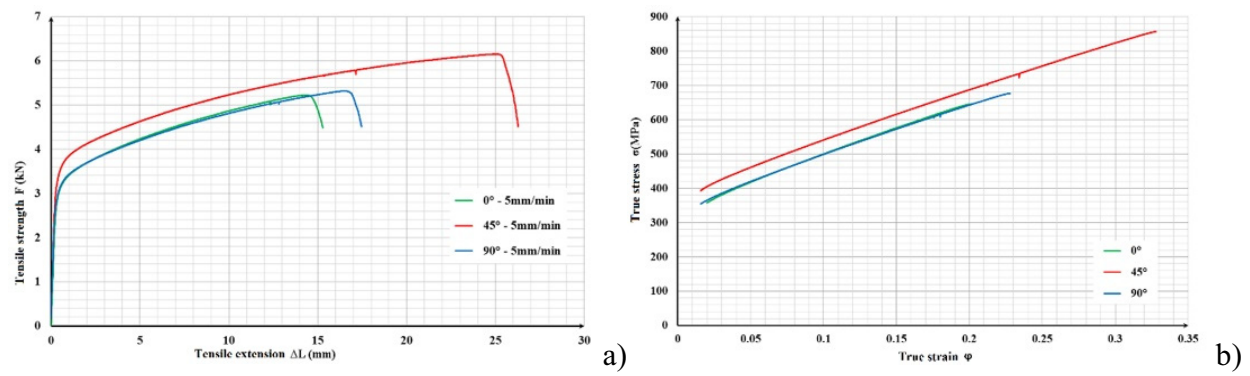

Fig. 2. Representative indicator diagrams of $\mathrm{X} 5 \mathrm{CrNi} 18-10$; $\mathrm{t} 0=0.8 \mathrm{~mm}$ : a) tensile strength - tensile extension, b) true stress - true strain.

As the load $\mathrm{F}$ and the elongation $\Delta \mathrm{L}$ depend on the shape and dimensions of the tested pieces, quantitative comparison of the mechanical properties of the material according to the tensile strength - tensile extension diagram is not possible. Therefore, the $F=f_{1}(\Delta L)$ diagram (Fig. 2 a) is converted into true stress - strain diagram $\sigma=f_{2}(\varphi)$, Fig. $2 b$. The resultant curves are parallel and reconcile one another with the strain increase. The three curves vary in their maximal strain and stress before the break. The true stress maximal values occur in $45^{\circ}$ cut samples $-\sigma=856.7 \mathrm{MPa}$ that is $27 \%$ and $33 \%$ higher than the $90^{\circ}$ and $0^{\circ}$ tested samples, respectively. The true strain of the $45^{\circ}$ cut samples $(\varphi=0.328)$ exceeds that of the samples cut at $90^{\circ}$ and $0^{\circ}$ directions with $44.5 \%$ и $51.6 \%$, respectively.

It is worth noting that the established variances in the mechanical properties data within the $0^{\circ}, 45^{\circ}$ and $90^{\circ}$ cut samples could be due to the manufacturing conditions and processing during the metallurgical process. Additionally, the non-homogenous composition and defects in the structure play essential role in the initiation of breaking and its dissemination during loading of the sheet material. The various types of defects including their nature, form, size and distribution could be the reason for the material breaking at different stress values.

To clarify and confirm the differences established from the applied stress, Vickers hardness measurements with a develop method [10] is used. The results of the average hardness values of the planes I, II and III of the undeformed and TT samples cut at $0^{\circ}, 45^{\circ}$ and $90^{\circ}$ angle to the RD are listed in Excel tables and graphically shown in Fig. 3. The ten measurements are averaged for each plane (I, II and III) of a tested specimen. As the number of the samples examined is six, the total number of the completed tables is equal to eighteen.

The comparative analysis of all planes of the samples reveals that the hardness increases irregularly for the different planes after the tensile test the samples. The hardness change of the $0^{\circ}$ cut sample is slight in contrast to the other $\left(45^{\circ}\right.$ and $\left.90^{\circ}\right)$ tensile tested samples. In plane I of the TT $0^{\circ}$ sample the hardness increase is $47.42 \%$ higher than the undeformed 
plane I and for plane II and III these percentages are $52.51 \%$ and $68.44 \%$, respectively. The reason for the higher hardness increase in plane II and III could be found in the favourable loading direction and the initial texture of the rolled material that could lead to grains fragmentation and lattice reorientation of the crystal lattice in these two planes. However, the $0^{\circ}$ cut sample is incapable of being highly deformed and strengthened because of the exhausted ductility in this direction.

The hardness increase in plane III of the $45^{\circ}$ cut sample is the highest $-107.44 \%$ and for plane I and II is $66.89 \%$ and $71.40 \%$ higher than the undeformed planes, respectively. These facts could be attributed to the favourable lattice reorientation and rotation of the grains in longitudinal direction and trans-crystal displacements in planes II and III. The maximal strain values set out in this TT sample is confirmed by the hardness test.

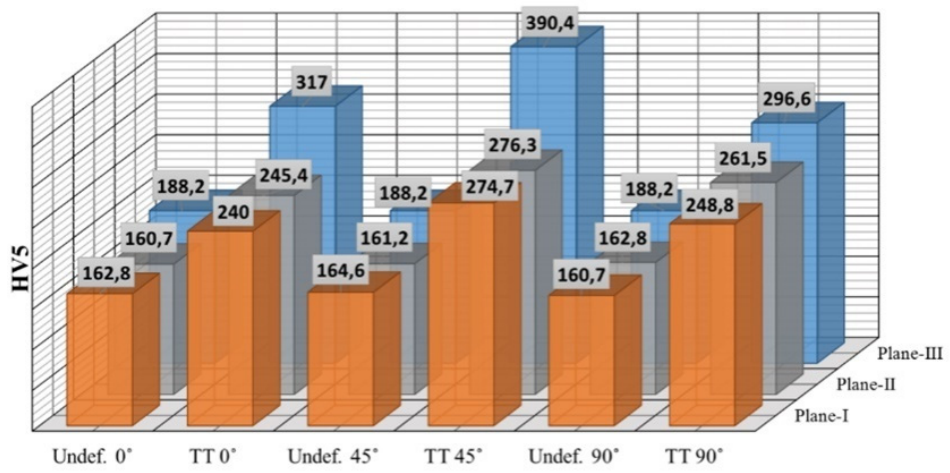

Fig. 3. Average hardness values before and after the tensile test of $0^{\circ}, 45^{\circ}$ and $90^{\circ}$ cut samples measured at planes I, II and III.

The increase in hardness of the $90^{\circ}$ cut sample is over $50 \%$ applied to the undeformed planes $-54.82 \%$ for plane I, $60.63 \%$ for plane II and $57.60 \%$ for plane III. The relatively balanced hardness change is due to the hindered and therefore, slight lattice reorientation and rotation of the initially textured structure, located normal to the direction of loading. Compared to the TT $0^{\circ}$ cut sample, the hardness and maximum strain values of the crosssection to the RD cut samples are higher.

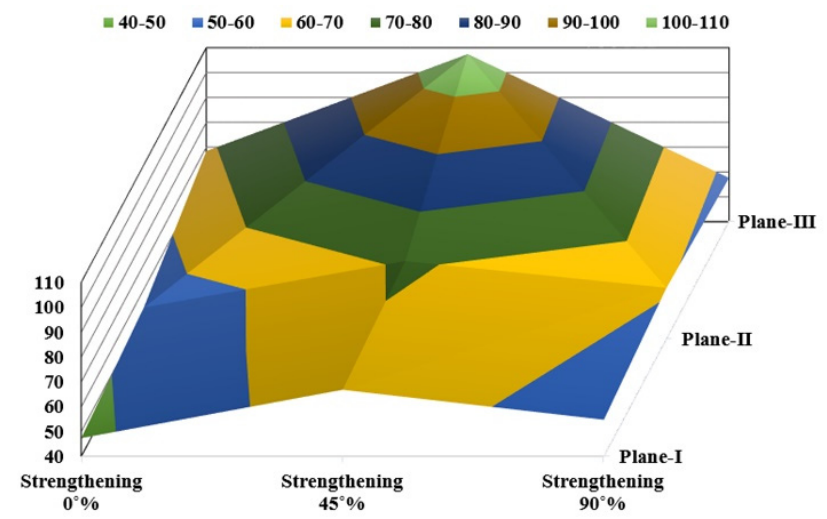

Fig. 4. Strengthening in percentage of the tensile tested samples $\left(0^{\circ}, 45^{\circ}\right.$ and $\left.90^{\circ}\right)$ applied to the undeformed samples for planes I, II and III. 
From the former hardness values, a summary diagram of the strengthening in percentage in the three planes (I, II and III) of the three $\left(0^{\circ}, 45^{\circ}\right.$ and $\left.90^{\circ}\right)$ cutting directions samples is shown on Fig.4. It could be noted that the major change in the strengthening is observed in plane III of $45^{\circ}$ cut sample - around $107 \%$. The slightest change in the strengthening percentage is found in plane I of $0^{\circ}$ cut samples - roughly $47 \%$. The summary diagram received enables a faster analysis of the favourable directions for deformation and consequent strengthening of the sheet materials during more complex loading schemes. Therefore, using Vickers hardness measurements, the strain values of a sheet metal could be determined and measures could be taken to avoid undesirable effect (such as local thickness reduction, delayed cracking, folding, etc.) during tensile of the materials.

The real microstructure images of each of the three planes of the six examined samples (three undeformed and three TT samples) assembled in cubes for a better visualization is presented in Fig. 5. In general the morphology of both planes (I and II) of the undeformed samples develop deformation texture of the previous rolling. In case of austenitic sheet steel the texture development is rather complex and depends on the direction of cutting of the samples. The dominating direction of the austenite texture corresponds to the rolling direction. With increasing the deformation degree the austenite undergoes stretching and the result of this depends on the cutting direction of the samples.

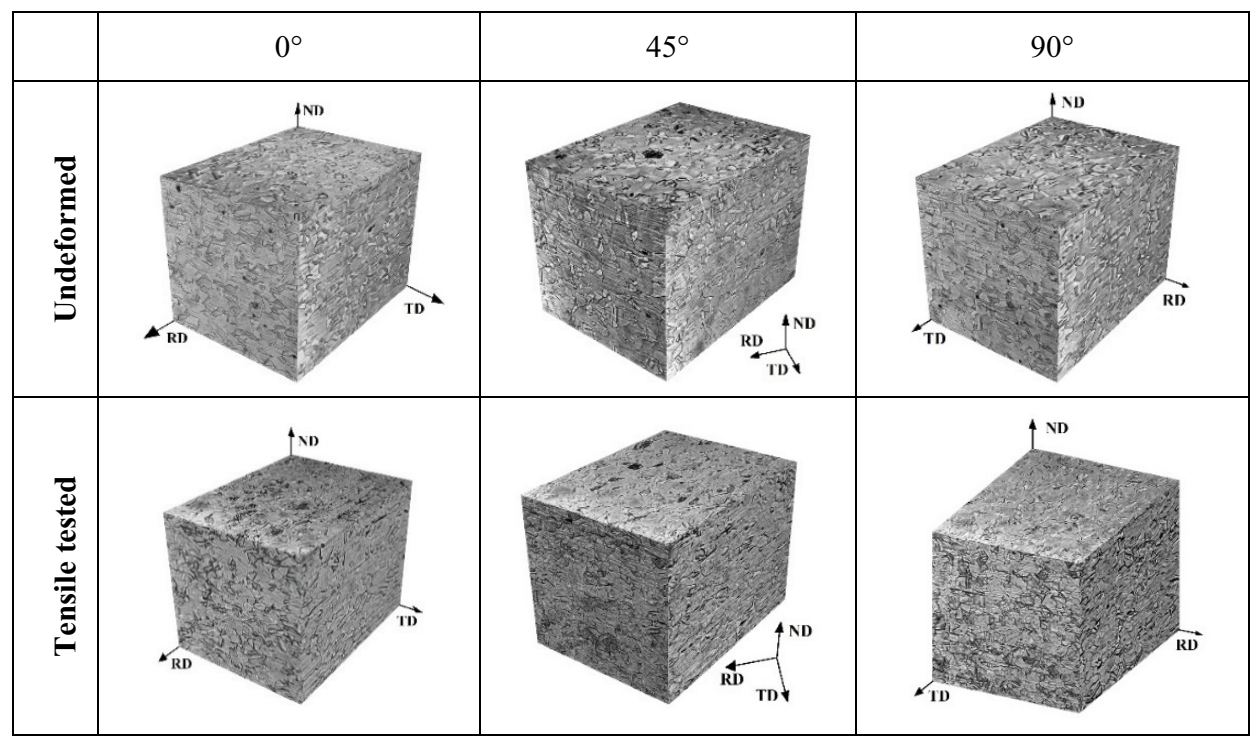

Fig. 5. Assembled 3D images of the microstructure in planes I, II and III of undeformed and tensile tested samples.

For more precise quantitative assessment of the results from the microstructure analysis, software processing of the images is used. The results obtained are shown in Fig. 6 and summarized in Table 2.

The main outcomes from the comparative microstructural analysis could be stated as follow: In the $0^{\circ}$ cut sample the grains are slightly fragmented in the planes I and II at the expense of reorientation. In plane III the grains are elongated and fragmented because of inter-crystal creep. The grains form is insignificantly changed but there is a strong reorientation tendency in plane III and especially in plane I. The tendencies observed are consistent with the hardness values measured at planes I and II. 


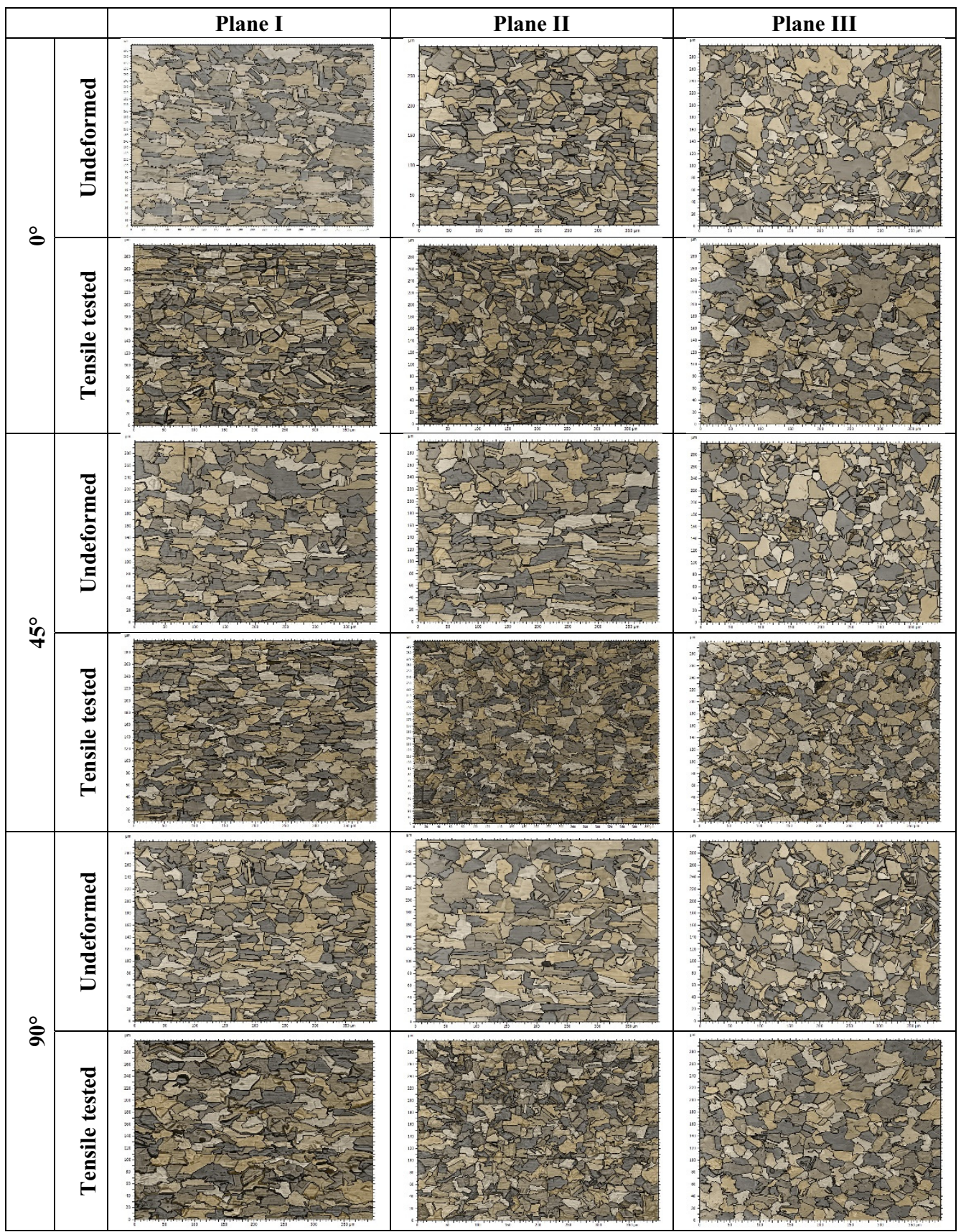

Fig. 6. A comparison of the software processed microstructures of the undeformed against tensile tested samples in planes I, II and III: a) $0^{\circ}$ cut sample; b) $45^{\circ}$ cut sample; c) $90^{\circ}$ cut sample.

In the $45^{\circ}$ cut sample there is a clearly visible grain fragmentation in all of the analysed planes confirmed by the increased number of grains, neighbours and the decrease in the mean diameter. The highest fragmentation is observed in plane II while the highest lattice reorientation is seen in plane I. This has a powerful effect on the surface roughness and 
hardness where the latter has the highest values (Fig. 3). The grains in the planes I and II have approximately equal form and the established reorientation in all three planes has lower values than that of the $0^{\circ}$ cut sample. The lattice reorientation in the $45^{\circ}$ cut sample after the tensile test is due to the pronounced grains fragmentation and trans-crystal displacement.

Table 2. Mean values of some microstructure determined parameters.

\begin{tabular}{|c|c|c|c|c|c|c|c|c|c|}
\hline \multicolumn{2}{|c|}{ Sample } & $\begin{array}{l}\text { No of } \\
\text { motifs }\end{array}$ & $\begin{array}{l}\text { Area, } \\
{\left[\mu \mathrm{m}^{2}\right]}\end{array}$ & $\begin{array}{c}\text { № of } \\
\text { neighbors }\end{array}$ & $\begin{array}{c}\text { Equivalent } \\
\text { diameter, } \\
{\left[\mu \mathrm{m}^{2}\right]}\end{array}$ & $\begin{array}{c}\text { Mean } \\
\text { diameter, } \\
{\left[\mu \mathrm{m}^{2}\right]}\end{array}$ & $\begin{array}{l}\text { Form } \\
\text { factor }\end{array}$ & $\begin{array}{l}\text { Round- } \\
\text { ness }\end{array}$ & $\begin{array}{l}\text { Orientati } \\
\text { on }\left[{ }^{\circ}\right]\end{array}$ \\
\hline \multirow{2}{*}{$\begin{array}{l}\text { Undef. } \\
0^{\circ}\end{array}$} & Plane I & 310 & 386 & 5.90 & 20.6 & 17.4 & 0.276 & 0.423 & 108 \\
\hline & Plane III & 505 & 237 & 5.64 & 15.5 & 14.1 & 0.340 & 0.441 & 90.7 \\
\hline \multirow{2}{*}{$\begin{array}{l}\text { TT } \\
0^{\circ}\end{array}$} & Plane I & 410 & 291 & 5.59 & 18.5 & 15.1 & 0.258 & 0.410 & 96 \\
\hline & Plane II & 448 & 267 & 5.53 & 17.7 & 15.1 & 0.280 & 0.498 & 93.3 \\
\hline \multirow{2}{*}{$\begin{array}{l}\text { Undef. } \\
45^{\circ}\end{array}$} & Plane II & 211 & 566 & 5.55 & 26.0 & 21.0 & 0.225 & 0.399 & 99.3 \\
\hline & Plane III & 583 & 205 & 5.71 & 14.8 & 13.7 & 0.348 & 0.464 & 93.6 \\
\hline \multirow{3}{*}{$\begin{array}{l}\mathrm{TT} \\
45^{\circ}\end{array}$} & Plane I & 372 & 321 & 5.61 & 19.4 & 16.1 & 0.245 & 0.519 & 86.9 \\
\hline & Plane II & 500 & 239 & 5.62 & 16.8 & 13.8 & 0.248 & 0.472 & 93.2 \\
\hline & Plane III & 468 & 255 & 5.65 & 17.2 & 15.2 & 0.289 & 0.482 & 89.1 \\
\hline \multirow{2}{*}{$\begin{array}{l}\mathrm{TT} \\
90^{\circ}\end{array}$} & Plane II & 404 & 296 & 5.60 & 18.7 & 15.4 & 0.251 & 0.710 & 88.3 \\
\hline & Plane III & 299 & 400 & 5.58 & 21.4 & 19.0 & 0.286 & 0.499 & 93.9 \\
\hline
\end{tabular}

In the $90^{\circ}$ cut sample the grains in plane I are enlarged and change their elongated form almost without reorientation. In plane II the grains are highly fragmented and change their roundness becoming more spherical. The grain enlargement could be detected also in plane III. The measured reorientation of the grains in planes II and III is low, although the former is higher than the same in plane I. The results from the microstructure of the sample are confirmed by the higher hardness values of planes II and III applied to plane I.

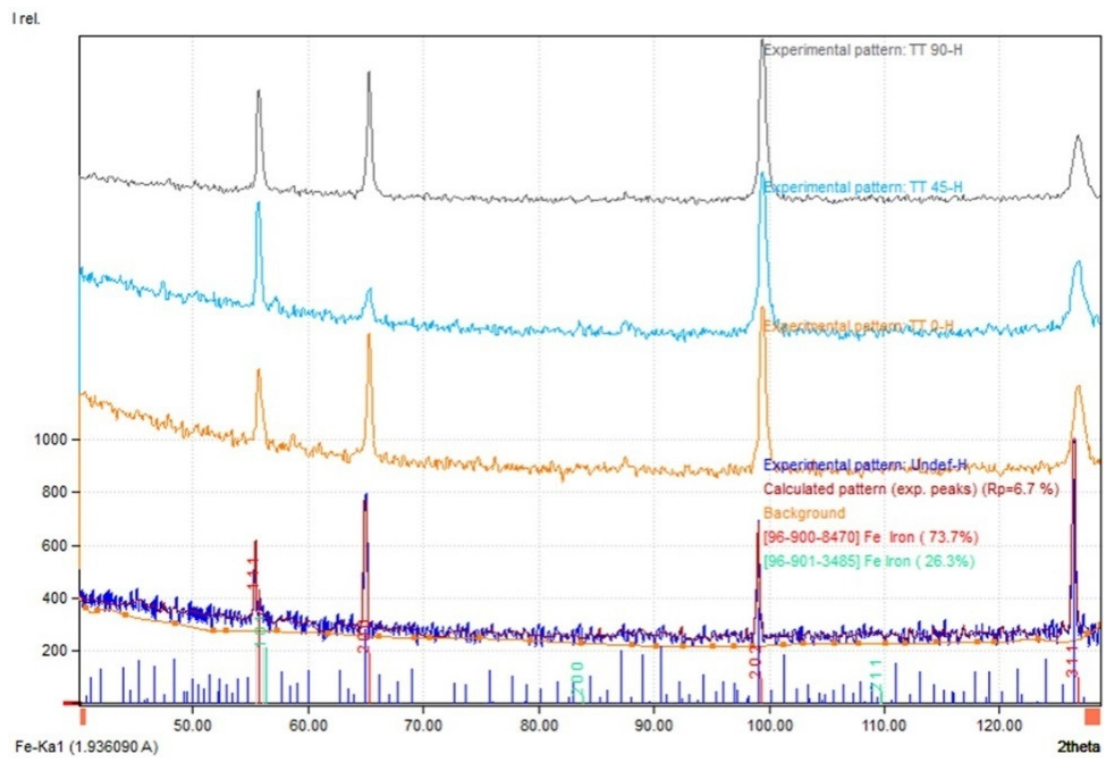

Fig. 7. Diffraction patterns of $\mathrm{X} 5 \mathrm{CrNi18}-8$ steel in initial state and after tensile test of the samples in horizontal direction of plane III; Bragg-Brentano geometry (BB). 
The statistically representative volume elements of the microstructure analysis are less than determining average macroscopic properties (strain localized pattern) but these shortrange characteristics shows a good consistency in the hardness measurements.

On the diffraction patterns of the steels in the initial and tensile tested state performed by Bragg-Brentano method (Fig. 7 and 8), reveal four strong diffraction lines from the planes (111), (200), (202) and (311) of austenite with low peak-to-peak noises. Additionally, week diffraction peaks from diffraction line (110) of $\alpha^{\prime}$-martensite are observed in the TT and initial state. The small quantity of the martensite phase detected in the undeformed state probably is a result of the cutting and subsequent preparation of the material for the investigations.

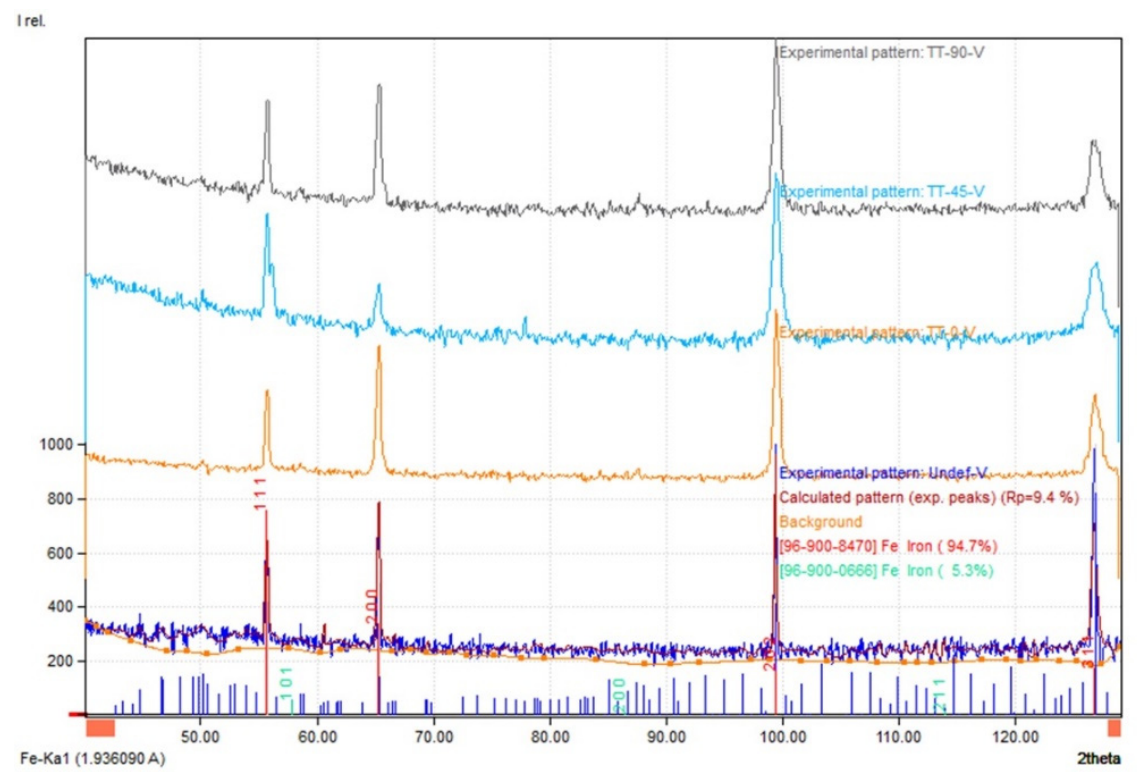

Fig. 8. Diffraction patterns of $\mathrm{X} 5 \mathrm{CrNi} 18-8$ steel in initial state and after tensile test of the samples in vertical direction of plane III; Bragg-Brentano geometry (BB).

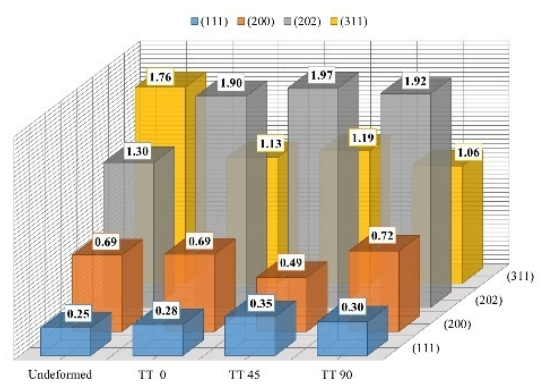

a)

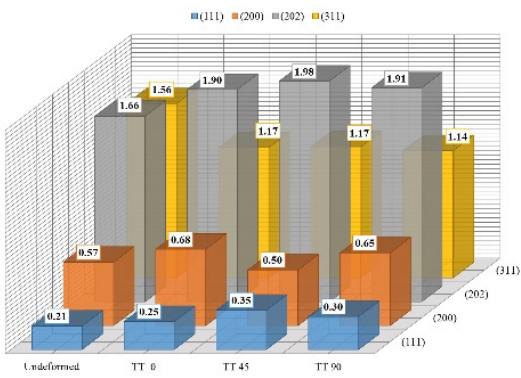

b)

Fig. 9. Calculated texture coefficients of the undeformed and TT samples measured at planes III in: a) horizontal direction; b) vertical direction.

The results of the calculated TC (Fig. 9) presents that the undeformed sheet material is strongly textured in (311) plane both in horizontal and vertical direction of scanning. Differences are detected in the (202) plane where the TC varies, which means that this plane doesn't have strong texture character. After the tensile tests the calculated changes in 
the TC are very much in line with that of the intensity changes measured at planes III and shown in Fig. 10.

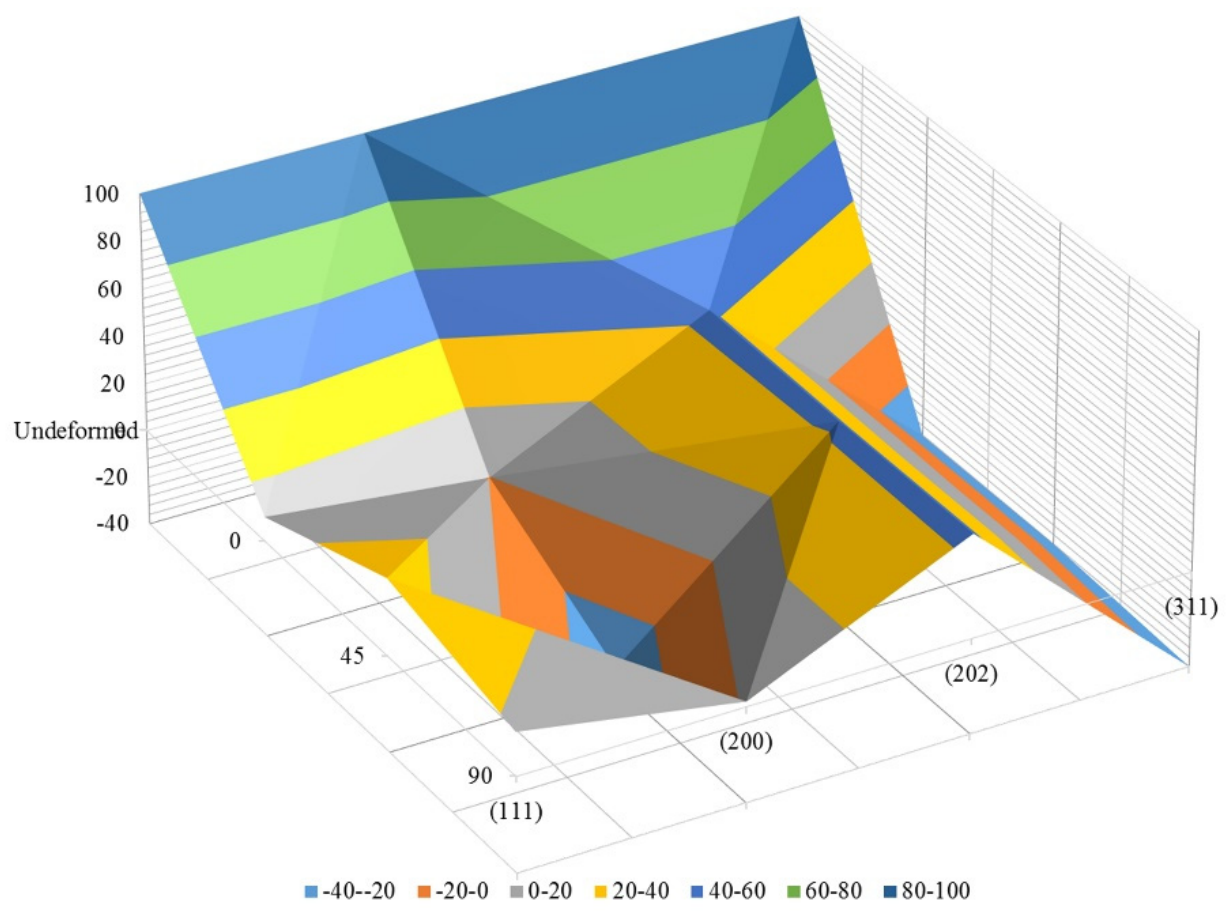

Fig. 10. Intensity changes (in percentage) of TT $0^{\circ}, 45^{\circ}$ and $90^{\circ}$ applied to the underformed sample measured at planes III in horizontal direction.

In the horizontal direction the largest is the increase in the intensity of (202) and the highest is the decrease in (311) lattice planes. Each reflection peak corresponds to a specific family of grains and its intensity is proportional to the contribution of the number of grains of the family in the irradiated volume. Therefore, the tensile test affects the variations among the individual planes and the lattice strain and the specific grain population orientations. In this connection, after the steel deformation the following main changes in the diffraction patterns intensities could be summarized as follow:

For the $0^{\circ}$ cut and TT sample the movements of the crystallographic planes show reorientation in austenite $(111)_{\gamma}$ and especially in $(202)_{\gamma}$ planes $(44.6 \%$ increase) at the expense of decrease of $(311)_{\gamma}$ plane intensity ( $36.3 \%$ decrease). In this case the creep of the material is not in the most favorable closed packed $(111)_{\gamma}$ plane, but mainly in $(202)_{\gamma}$ one. The results observed explains the earlier rupture of the sample because of the less ductility of the $(202)_{\gamma}$ plane. As a percentage applied to the undeformed sample the intensity increase in $(202)_{\gamma}$ plane is $44.6 \%$ and only $10.35 \%$ for the $(111)_{\gamma}$ plane.

All of the examined planes of $45^{\circ}$ cut TT sample go through substantial changes in the intensities. The latter increases in $(111)_{\gamma}$ and $(202)_{\gamma}$ planes with $32.63 \%$ and $43.28 \%$, respectively, while $(311)_{\gamma}$ and $(200)_{\gamma}$ lines become weaker (36 and $33.22 \%$ decrease, respectively). It follows that the crystals in the $45^{\circ}$ cut sample has had a favorable initial position applied to the load direction thus contributing to the balanced reorientation in the crystallographic planes. This allows for $51.6 \%$ higher degree of deformation of this sample as compared with $0^{\circ}$ cut TT sample.

In the $90^{\circ}$ cut TT sample the intensity of the $(111)_{\gamma}$ plane increases with $18.36 \%$, but simultaneously the creep occurs in $(202)_{\gamma}$ plane $(43.58 \%$ increase). The reorientation in both 
planes is for the account of $(311)_{\gamma}$ which intensity decreases with $41.7 \%$. In contrast to $45^{\circ}$ cut sample, the $(200)_{\gamma}$ increases slightly with $2.19 \%$.

Weak martensite (101) $\alpha^{\prime}$ - lines are found in most of the tensile tested samples. The diffraction lines of $\varepsilon$-martensite are not disclosed that proves the direct $\gamma \rightarrow \alpha^{\prime}$ transformation in this steel in such test conditions. As determined from the XRD results, the tensile test induces some transformation of the metastable austenite in magnetic $\alpha^{\prime}$ martensite. For that reason SEM analysis is performed and some of the results are shown in Fig. 11. The SEM observations lead to the assessment that more martensite is observed in cross-sections (plane II) of all samples but even in that plane, its quantity is small, as noted also by the XRD analysis.

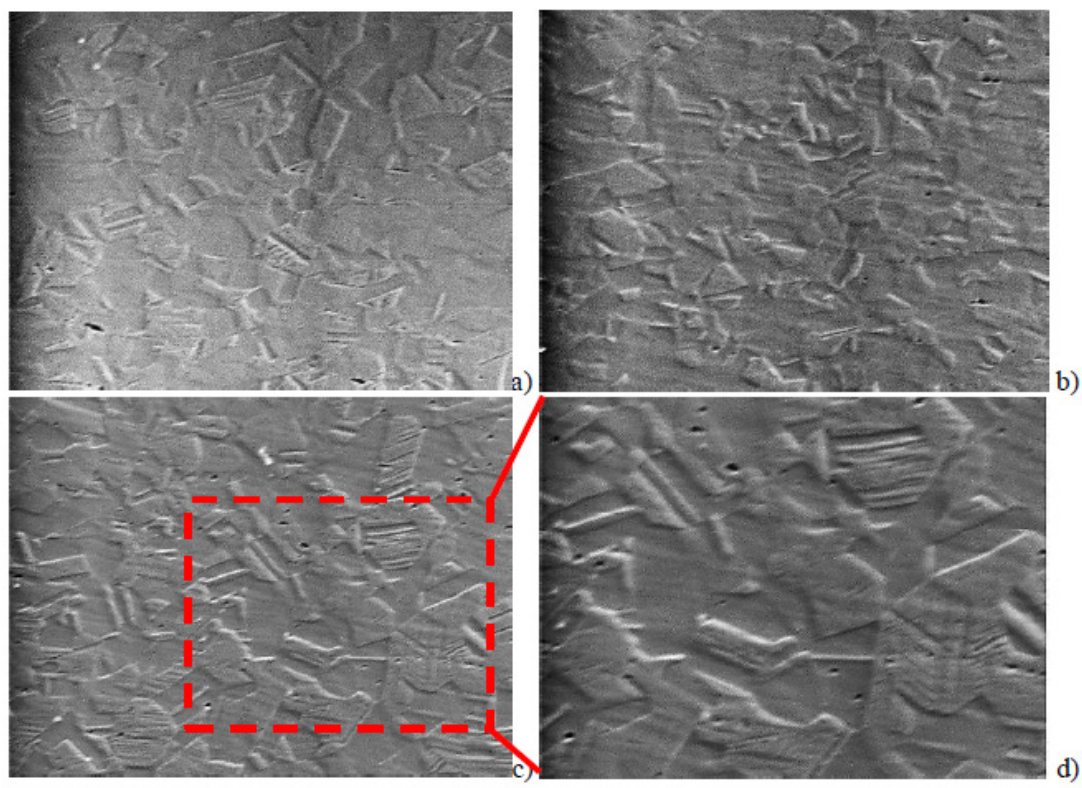

Fig. 11. Secondary image micrographs of plane II structure: a) $0^{\circ}$ cut TT sample; Magn.600×; b) $45^{\circ}$ cut TT sample Magn. 600×; c) $90^{\circ}$ cut TT sample: Magn.600×; d) 90 cut TT sample: Magn. 1000×.

During the deformation, weak magnetic properties are detected as soon as $14 \%$ of deformation is reached and the former increase with the increase of the degree of deformation. This suggests that structure changes occur in the crystal lattices of the samples cut at different directions. The presence of $\alpha^{\prime}$-martensite, as well as the increased dislocation density will lead not only to the observed change in the magnetic permeability, but also to enhance work hardening because of the dislocation accumulation at the austenite/martensite interfaces [11]. In contrast to the assumption made by J. Talonen at al. [12] that the martensite form a "continuous network" trough the material, as seen in Fig. 11, in none of the samples the $\alpha^{\prime}$-phase does not form such a network that could effectively impede the flow of the soft austenite.

\section{Conclusions}

The uniaxial tensile test deformations at macro- and microscopic scale for an austenitic steel sheet has been examined using hardness tests, microstructure and XRD analysis. The proposed methodology for examination of an atypical behaviour of sheet metal materials clarifies the reasons why anirregular degree of deformation is observed in the three 
different angles of cutting. The Vickers hardness measurement is one step leading to the determination of the changes in plasticity with the material strengthening. The use of XRD analysis provides the opportunity to identify the causes of the higher plasticity in a certain directions of the austenitic sheet material. The analysis and equipment of this kind is far cheaper than other modern methods of testing (such as automated crystallographic orientation indexation in a TEM (ACOM-TEM), electron backscattered diffraction (EBSD), etc.). The identification of the changes of the crystallographic planes on the basis of the deformation scheme is a sufficiently accurate, reliable and proved over time method. The surveys carried out in this paper, show that the XRD analysis is also suitable for the determination of the anisotropy of the material. The differences in the relative intensities of the reflection lines compared to the theoretical intensity of each austenite peak indicate that

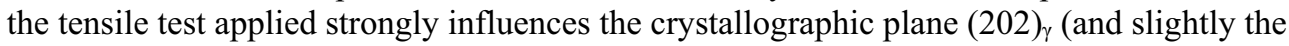
most favourable $(111)_{\gamma}$ ) at the expanse of the reduce in the intensity of the textured $(311)_{\gamma}$ one. This study also indicates that the grains fragmentation and austenite deformation take precedence over the martensite transformation during the uniaxial tensile test of the sheet material. The deployment of modern software products for quantitative microstructure analysis allows for rapid identification of the changes in grains shape, dimensions, orientation, fragmentation and other parameters.

The proposed examination approach with several alternative methods for analysis of the non-uniform deformation of an anisotropic sheet material will be verified for other relevant materials and other loading schemes of deformation. In that way the assurance and reliability of the proposed examination methods for analysis will be confirmed or infirmed.

The paper reflects the outcomes of the project № 16 - FMME - 02, financed by the Fund „Scientific Research" of University of Ruse "A. Kanchev".

\section{References}

1. J. Slota, M. Jurčišin, E. Spišák, M. Tomáš, M. Šiser, Acta Metall Slovaca, 21 4, 269277 (2015)

2. Das A., P. C. Chakraborti, S. Tarafder, H. K. D. H. Bhadeshia, J Mater Sci Tech, 271 366-369 (2011)

3. D.-F. Li, Noel P. O'Dowd, C. M. Davies, S.-Y. Zhang, European J of Mech - A/Solids 30 (5) 748 (2011)

4. N. Habibi, A. Zarei-Hanzaki, H.-R. Abedi, J Mater Process Technol 224 102-116 (2015)

5. N. Afrin, D.L. Chen, X. Cao, M. Jahazi, Mater Sci Eng A 472 179-186 (2008)

6. M. Baig, E. El-Danaf, M. Soliman, A. Almajid, W. Alshalfan, and Khaled Alhajeri, Mater Testing 57,10, 897-903 (2015)

7. J. Talonen, P. Nenonen, G. Pape, H. Hänninen, Metall Mater Trans A, Vol. 36A 421 (2005)

8. Class 26_handout MTSE 30102013 Supplemental Texture \& Grain Size Calculation.pdf, Polycrystallography, Institute of North Texas, http://engineering.unt.edu/materials/sites/default/files/sites/default/files/lamma/oldsite/ documents/Class26_handout $\% 20$ MTSE\%203010\%202013\%20SUPPLEMENTAL \%2 0TEXTURE\%20\% $26 \% 20$ GRAIN\%20SIZE\%20CALCULATION.pdf, (2013)

9. Yankov E., Sheet materials testing using biaxial hydraulic bulge test (Dissertation, Ruse, 2014)

10. YankovE., D. Gospodinov, V. Gagov, Scientific conference RU \& SU'14, 53 (2014)

11. T. Narutani, Mater. Trans. JIM, 30, 33-45 (1989)

12. J.Talonen, P. Nenonen, G. Pape, H. Hänninen, Metall Mater Trans A 36A, 421 (2005) 